\title{
Filigrane
}

Écoutes psychanalytiques

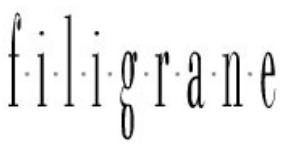

\section{Le roman familial psychanalytique d'Anna Freud et le nôtre. Filiation et homosexualité}

\section{Marie Hazan}

Volume 28, numéro 1, 2019

URI : https://id.erudit.org/iderudit/1064602ar

DOI : https://doi.org/10.7202/1064602ar

Aller au sommaire du numéro

\section{Éditeur(s)}

Santé mentale et société

ISSN

1192-1412 (imprimé)

1911-4656 (numérique)

Découvrir la revue

Citer cet article

Hazan, M. (2019). Le roman familial psychanalytique d'Anna Freud et le nôtre. Filiation et homosexualité. Filigrane, 28(1), 149-168.

https://doi.org/10.7202/1064602ar

\section{Résumé de l'article}

Anna Freud, la sixième et la plus jeune des enfants de Sigmund Freud, est devenue l'héritière et la gardienne officielle de l'oeuvre de son père, quoique de manière ambiguë et dans la controverse. Fondatrice de la psychanalyse d'enfants avec ou contre Melanie Klein, elle a aussi cofondé des écoles mettant de l'avant une pédagogie psychanalytique et novatrice, non sans révéler ce faisant des interférences entre son analyse, son oeuvre et sa vie. Son analyse avec son père, qui l'appelait " son Antigone ", a fait l'objet d'articles de Freud ( Un enfant est battu » en 1919) et d'elle-même (" Fantasme d"“être battu” et rêverie » en 1922). Publiés très tôt, ces textes suscitent ambivalences et critiques voilées, ainsi que des interprétations sauvages selon lesquelles Anna Freud était tour à tour vieille fille et lesbienne. Cet article interroge les liens entre son analyse, poursuivie sous une élection paternelle dont elle bénéficie tout en étant prisonnière, et le regard que nous portons sur elle aujourd'hui. Si Anna Freud a certes été fidèle à la mémoire et à l'oeuvre de son père et à ce qu'elle aura cherché à en protéger, elle a également eu une longue vie après la mort de celui-ci, vie où elle a aimé et frayé sa propre voie, notamment en fondant des lieux thérapeutiques psychanalytiques pour les enfants et en créant une oeuvre originale en psychanalyse.
Ce document est protégé par la loi sur le droit d'auteur. L'utilisation des services d’Érudit (y compris la reproduction) est assujettie à sa politique d'utilisation que vous pouvez consulter en ligne.

https://apropos.erudit.org/fr/usagers/politique-dutilisation/ 


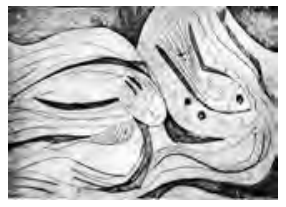

\title{
Le roman familial psychanalytique d'Anna Freud et le nôtre. Filiation et homosexualité
}

\author{
Marie Hazan
}

\begin{abstract}
Résumé: Anna Freud, la sixième et la plus jeune des enfants de Sigmund Freud, est devenue l'héritière et la gardienne officielle de l'œuvre de son père, quoique de manière ambiguë et dans la controverse. Fondatrice de la psychanalyse d'enfants avec ou contre Melanie Klein, elle a aussi cofondé des écoles mettant de l'avant une pédagogie psychanalytique et novatrice, non sans révéler ce faisant des interférences entre son analyse, son œuvre et sa vie. Son analyse avec son père, qui l'appelait «son Antigone», a fait l'objet d'articles de Freud («Un enfant est battu» en 1919) et d'elle-même ("Fantasme d'“être battu” et rêverie» en 1922). Publiés très tôt, ces textes suscitent ambivalences et critiques voilées, ainsi que des interprétations sauvages selon lesquelles Anna Freud était tour à tour vieille fille et lesbienne. Cet article interroge les liens entre son analyse, poursuivie sous une élection paternelle dont elle bénéficie tout en étant prisonnière, et le regard que nous portons sur elle aujourd'hui. Si Anna Freud a certes été fidèle à la mémoire et à l'œuvre de son père et à ce qu'elle aura cherché à en protéger, elle a également eu une longue vie après la mort de celui-ci, vie où elle a aimé et frayé sa propre voie, notamment en fondant des lieux thérapeutiques psychanalytiques pour les enfants et en créant une ouvre originale en psychanalyse.

Mots clés: Anna Freud; psychanalyse; enfants; pédagogie psychanalytique; transmission; homosexualité.
\end{abstract}

\begin{abstract}
Anna Freud is Sigmund Freud's sixth and youngest child. She became the caretaker of the psychoanalytical legacy and founded, with or against Melanie Klein, child psychoanalysis. However, all of this did not occur without ambiguity and controversy. She was in analysis with her father, and in 1922 she wrote a paper about her fantasies, just as Freud did before (A Child Is Beaten, 1919). She was secretly labeled and criticized as an old maid and as a lesbian. The link between her analysis with Freud and her being chosen to represent psychoanalysis was publicly questioned, as she was caught in this legacy. Did she sacrifice her own life and sublimate her libido for him, therefore becoming his "Antigone," as he named her? In this paper, I analyze our gaze on her in regard to the paradox between love in reality and faithfulness to memory. Although her attachment to her father's legacy deprived her in a way, recent papers show how she lived a long life after his death, a life in which she loved, created and found her own path.
\end{abstract}


Key words: Anna Freud; psychoanalysis; children; psychoanalytical pedagogy; transmission; homosexuality.

Le transfert n'est [...] qu'un fragment de répétition et la répétition est un transfert du passé oublié. [...] Privé de mémoire, l'analyste répète l'acte de fondation de l'analyse, sans savoir qu'il s'agit d'une répétition. (Schneider, 1980, p. 29-30)

$\checkmark$

'ai longtemps éprouvé une affection spéciale et une grande curiosité pour la jeune Anna Freud, personnage romanesque s'il en fut. L'image de cette jeune fille passionnée contraste avec celle plus austère de la vie qu'elle eut à Londres suite à la mort de son père. Elle est la dernière et presque «fille unique» de Freud, comme il aimait à le lui dire, et ce dernier l'a élue - non sans ambiguïté - pour transmettre et poursuivre son œuvre: elle fut ainsi la porte-parole et la gardienne parfois intransigeante du temple; à la fois vestale, Antigone et fondatrice, tout en tissant des liens étroits avec un environnement familial par extension. Elle inspire cependant des questionnements et des réactions plus ou moins vives, soupçonneuses, obscures ou ambivalentes. Anna Freud est un personnage à visages et histoires multiples et suscite en retour des réactions transférentielles diverses. Mettre en lumière son évolution et ce qui a amené Freud à la nommer héritière officielle au détriment de ses fils, de ses collègues et disciples alimente également des interrogations.

Projections paradoxales sur l'histoire de la psychanalyse et ses fondateurs, les images d'Anna Freud sont contrastées et controversées. Certaines représentations, prononcées à mi-voix, sont peu flatteuses : celle d'une jeune fille rangée, rigide et ennuyeuse, ou encore celle d'une vieille fille sévère, trop attachée au souvenir de son père, et qui serait morte vierge ${ }^{1}$. Anna Freud représente l'orthodoxie obligée et la protection acharnée des secrets psychanalytiques, plus spécialement ceux de Freud, qu'elle a jalousement gardés.

Concernant la vie sexuelle qu'elle n'aurait pas eue, ayant sublimé ses pulsions et pratiqué et prôné la «cession altruiste» selon ses propres mots et ceux de Sigmund Freud, il y aurait beaucoup à dire. Le "cas clinique» qu'elle présente le 31 mai 1922 afin de devenir membre de la Société psychanalytique de Vienne (WPV) (Roudinesco, 2012, p. 14), et qui serait une version aisément reconnaissable d'elle-même, a fait l'objet de plusieurs commentaires (Young-Bruehl, 1988, p. 117). Exposés à tous, ces fragments de sa 
propre étude de cas remettent cependant en question sa réputation d'être pudique et réservée.

Plus récemment, cette image désuète d'une vieille fille fidèle à son père a d'ailleurs été remplacée par une nouvelle, remaniée au goût du jour: Anna Freud et Dorothy Burlingham auraient formé une famille homoparentale (Roudinesco, 2014 a; Menahem, 2003, p. 11; Drillon, 2012). Pourquoi pas? Certes, dans les biographies d'Anna, rien ne l'indique explicitement; le thème est plutôt évité et fait même l'objet de dénégations pendant très longtemps. Or, rien ne va à l'encontre de cette hypothèse ou plutôt de cette (ré) vision actuelle, indicative de notre transfert sur la vie des fondateurs... La transmission de la psychanalyse passerait-elle par des crises cristallisées sur nos transferts variables?

Ce qui était auparavant un secret (de polichinelle), frappé d'un interdit de dire et de penser - et donc motif à rumeurs et même à médisances -, est désormais énoncé avec simplicité et candeur: Dorothy Burlingham aurait été la compagne de vie d'Anna et elles auraient même entretenu une vie familiale. Pourtant, Anna, à l'instar d'Ernest Jones, mais contrairement à son père, condamnait l'homosexualité et la considérait comme une perversion et une maladie...

La supposée homosexualité - encore à définir - et vie conjugale d'Anna Freud serait-elle moins taboue aujourd'hui, d'autant plus que les protagonistes sont morts au siècle dernier? L'homosexualité est certainement plus admise aujourd'hui, de telle sorte que sa reconnaissance actuelle suscite une relecture du passé.

Il y a donc encore beaucoup à dire sur Anna Freud, et de nombreux écrits sont publiés régulièrement la concernant, que ce soit à propos de sa correspondance, de son legs, de sa théorie sur la psychanalyse d'enfants ou encore de son célèbre ouvrage Le Moi et les mécanismes de défense (1936). De plus, les écoles qu'elle a fondées à Vienne, puis à Londres, sont des lieux d'expression de sa vocation - à savoir l'articulation de la psychanalyse avec une mission pédagogique novatrice et progressiste, qu'elle souhaitait salvatrice - et constituent le témoin d'une mission accomplie pour et auprès des enfants.

J'ai délibérément choisi de centrer mon propos sur ses relations familiales au sens large, soit avec la famille Freud, avec Eva Rosenfeld et Dorothy Burlingham, avec les enfants des écoles qu'elles ont fondées et enfin avec la famille psychanalytique. J'y inclus de surcroît la question de son analyse avec son père, ainsi que ses trop nombreux deuils et l'ombre de la Shoah. 
Je laisserai donc de côté les conflits et les querelles institutionnelles avec Melanie Klein à propos de la psychanalyse d'enfants, et je ne traiterai pas (cela supposerait un travail en soi) des importants apports théoriques et institutionnels d'Anna Freud à la psychanalyse d'enfants et à la psychanalyse américaine.

\section{Anna Freud est un roman}

Anna Freud est un personnage hors du commun, qui a connu plusieurs vies. À commencer par sa curiosité pour la psychanalyse dès son jeune âge, sa fascination pour son père et ses travaux (elle campait littéralement derrière sa porte), son analyse avec lui, et ce jusqu'à la prise en charge de la personne, de l'âme et du corps de Sigmund Freud durant sa maladie, ses multiples opérations à la mâchoire et les dernières années de sa vie. Elle était à la fois sa porte-parole, son infirmière et sa protectrice tenace. Elle aura aussi fondé la psychanalyse d'enfants en Angleterre avec, ou plutôt contre, Melanie Klein. Tout un parcours de vie fascinant où se mêlent voyages, découvertes et renommée, longues amitiés et liens intenses dans un milieu psychanalytique alors en émergence et en ébullition; mais aussi tragédies, deuils et malheurs, ponctués par l'histoire du xx siècle dans la Mitteleuropa et la disparition d'un monde.

Anna Freud est née à Vienne au XIX ${ }^{e}$ siècle. Elle a voyagé en Europe durant les belles années, trop brèves, de sa jeunesse, avant la maladie de son père et la Deuxième Guerre; avant les deuils, l'exil, la mort de Freud. Avant d'élire domicile à Londres avec ses parents, elle est arrêtée par la Gestapo suite à l'Anchluss de $1938^{2}$, ce qui convainc son père d'enfin quitter Vienne, devenue terre hostile. S'ils ont réussi in extremis à fuir le nazisme, une partie de la famille, à commencer par quatre des cinq sœurs de Freud, a péri lors de la Shoah. Alfred Gottwaldt (2004) reconstitue la terrible histoire des quatre tantes d'Anna, dévoilée seulement après la guerre:

Avec ce train arrivaient Marie Freud (née le 22 mars 1819) [...], Adolfine Freud (née le 23 juillet 1862) [...] et Pauline Winternitz, née Freud (née le 3 mai 1864) [...], le jour suivant en Bohême [...] Par contre la quatrième sœur, Rosa Graf, née Freud (née le 31 mars 1860) [...], a été, parmi 956 personnes, déportée seulement deux mois plus tard, le 27 août 1942, avec le «transport des personnes âgées IV/9» de Vienne à Theresienstadt. Il s'agit là du $38^{e}$ transport de Juifs de Vienne. (Gottwaldt, 2004, p. 1310) 
Seule une des sœurs, nommée Anna Freud elle aussi, mariée avec Eli Bernays, le frère de Martha, a émigré aux États-Unis en 1892. Heureusement pour lui, Freud est mort sans avoir su que ses sœurs avaient connu cette fin tragique.

\section{Première scène primitive: Freud, Fliess, la psychanalyse et Anna}

Dernière des six enfants de Sigmund et Martha Freud, cadette de leurs trois fils et deux filles (l'aînée, Mathilde, avait 8 ans à sa naissance), Anna Freud est née le 3 décembre 1895. Son père se plaisait à répéter que, venue au monde en même temps que la psychanalyse, elle lui aurait donné bien plus de satisfaction... Selon l'expression d'Emilio Rodrigué (1996b), la psychanalyse et Anna sont jumelles.

Néanmoins, dans le rêve de l'injection faite à Irma, rêve inaugural de L'interprétation des rêves (Freud, 1900), la grossesse de Martha est signalée comme ayant été une gêne et un contretemps ${ }^{3}$. La légende veut aussi que l'annonce de la venue d'Anna ait été accueillie avec peu d'enthousiasme par ses deux parents. Anna, enfant délaissée, s'avère pressée de grandir, un peu rebelle, mais dévouée. Elle devient leur bâton de vieillesse, dédiée à son père bien sûr, mais aussi à sa mère et à la tante Minna pourtant jalousée, et même à sa famille élargie, notamment à ses neveux et leur père Max Halberstadt au moment du décès de Sophie, la sœur enviée, aimée et détestée, brutalement emportée en un éclair par la grippe espagnole en juin 1920. Elle passe plusieurs semaines à s'occuper des enfants et de Max, veuf et pour un temps inconsolable. Ce sont pour ainsi dire ses premiers patients: "ses "premiers malades" furent ses deux petits neveux orphelins» (Gay, 1988, p. 500). Elle a son préféré, l'aîné, Ernst, qu'elle aurait aimé adopter... Il devient son protégé pour la vie et elle l'accueille dans son école à Vienne après le décès de Sophie et de son fils Heinz, dit «Heinerle», né en 1918 et décédé en 1923 d'une pneumonie, deuxième mort tragique dans la petite famille en si peu de temps.

Juste avant la naissance d'Anna en 1895, Freud est lié à Wilhelm Fliess par une relation d'amitié intense, épistolaire surtout, amitié qui a fini par être orageuse et mener à une rupture définitive. Freud lui écrit de nombreuses lettres de 1887 à 1904 (les lettres de Fliess n'ont pas été retrouvées), où il confie ses sentiments et relate les événements de sa vie familiale et quotidienne. Il s'épanche longuement sur ses états d'âme et, surtout, il lui expose en primeur ses idées, réflexions et projets durant cette période de 
créativité extraordinaire et bouillonnante. L'influence - plutôt déplorée et décriée - sur Freud de Fliess, un otorhinolaryngologiste qui avait des idées fumeuses sur la sexualité et la «périodicité vitale» et qui établissait un lien entre le pénis et le nez, questionne et intrigue. Fliess s'avère avoir été un témoin, voire un passeur, de l'auto-analyse de Freud, qui lui écrit de très nombreuses lettres, ponctuées par quelques rencontres pompeusement baptisées «congrès». Plutôt que bon ami, Fliess est qualifié de «tyran» et de «mauvais génie» «ayant subi un meurtre d'âme» par Philippe Réfabert (2004, p. 146) qui éclaire de manière troublante cet aspect obscur de la naissance de la psychanalyse. Il souligne l'emprise que Fliess avait sur Freud - sa seule passion selon Ernest Jones -, à un moment où il traverse «une crise d'une fécondité inouïe» (Réfabert, 2004, p. 145) : «Fliess est avec Freud comme ce parent qui, pour survivre à sa mort psychique, ne lâche pas l'enfant dont il fait sa créature. Fliess recueille la pensée luxuriante de Freud. Il se fait le destinataire et le fidèle dépositaire de lettres sublimes» (p. 147). Freud manifeste dans ses confidences une soumission et un abandon étonnants; à cette mesure, la déception et le sentiment de trahison qui s'ensuivent sont immenses et cuisants. Freud aurait été séduit, subjugué, et ce dès la première rencontre... Dans Le scénario Freud (1984), Jean-Paul Sartre décrit Fliess avec « un beau visage démoniaque [...] aux grands yeux brillants et autoritaires». Rodrigué de conclure: «Autrement dit, ce serait un cas de cruauté constitutionnelle.» (Rodrigué, 1996a, p. 285)

Freud - et Anna elle-même - souhaitait que ces lettres soient détruites, tout comme Anna refusera plus tard que sa correspondance avec Eva Rosenfeld soit rendue publique. Même si la relation entre Freud et Fliess n'est pas du tout la même que celle entre Anna et Eva, ces deux relations épistolaires sont semblables dans leur spontanéité, leur ton intimiste, leur élan et la chaleureuse amitié témoignée, suivie dans les deux cas d'une grande déception. À la lumière de la vie sentimentale qu'elle aura avec Dorothy Burlingham, Anna aurait-elle été conçue dans ce climat d'amitié passionnelle? À ce titre, rappelons que, si le nouveau-né avait été un garçon plutôt qu'une fille, Freud l'aurait prénommé «Wilhelm». De plus, Robert Fliess, fils de Wilhelm, né le $1^{\text {er }}$ janvier 1896, soit quelques jours seulement après Anna, et donc en quelque sorte son jumeau conçu pendant cette même relation fusionnelle, est devenu aussi psychanalyste.

L'histoire d'amitié entre Freud et Fliess finira très mal: après quelques dénégations de la part de Freud, notamment à propos de l'erreur médicale commise par Fliess sur Emma Eckstein, et des accusations de plagiat, 
déplacées et choquantes, de la part de Fliess, ce sera la rupture brutale et définitive.

Petite, Anna détestait l'être: elle voulait tant être grande! Dernière de la fratrie à habiter avec ses parents et la tante Minna, elle s'occupe de tout, surtout de son père, de «l'essentiel et l'inessentiel» dans les mots de Freud.

Par la suite, supplantant sa mère et prenant toute sa place auprès de son père - qui la lui accorde avec joie -, abandonnant son métier d'institutrice pour lequel elle était fraîchement diplômée, elle devient son «Antigone» et sa «Cordélia» (Fendrik, 1989, p. 70-71; Rodrigué 1996b, p. 221), mais aussi son infirmière, sa porte-parole dans les congrès de psychanalyse au moment où il est affligé d'une horrible prothèse appelée «le monstre», sa traductrice et, ultimement, son administratrice et sa gestionnaire pour la maison psychanalytique. Psychanalyste d'enfants et fondatrice d'écoles et de lieux de vie à pédagogie psychanalytique (d'abord à Vienne en 1927 avec Eva Rosenfeld et ensuite avec Dorothy Burlingham), elle publie très tôt sur le sujet et fait partie de la famille psychanalytique. Elle est à la fois la lectrice et l'interlocutrice des travaux psychanalytiques de l'époque et l'analysante de son père.

Selon une formule d'Elisabeth Roudinesco, «elle n'est désirée ni par sa mère, ni par son père» (Roudinesco, 2012, p. 12). Par-delà l'ambivalence de Freud à son égard dès sa conception, elle sera pourtant soutenue par le désir de son père et promise à un destin marquant, surtout après le départ de ses frères et sœurs. On sait combien est complexe la notion de désir d'enfant! Il est donc difficile, connaissant la suite de sa vie et de sa carrière, de déclarer qu'Anna n'était pas désirée. Didier Anzieu, dans son analyse fouillée du rêve de l'injection faite à Irma (Anzieu, 1959, p. 39-68), rappelle les associations de Freud liées à la grossesse de Martha, à son sentiment de culpabilité vis-àvis de celle-ci et à son désir de fausse-couche, mais aussi à celui de cet enfant qui deviendra psychanalyste et s'appellera «Wilhelm» ou «Anna». Selon Didier Anzieu, l'épilogue du rêve, c'est qu'il assume l'œuvre et l'enfant: «De cette vie à venir, dont est grosse Martha, Freud se sent maintenant, comme de son œuvre, pleinement responsable» (Anzieu, 1959, p. 51).

Autour de son berceau, la fée avec sa baguette magique a énoncé la prophétie: elle sera psychanalyste comme son père et gardienne du temple; elle lui succédera sur le trône. Mais la fée Carabosse décrète aussi: elle ne se mariera jamais et restera fidèle à son père! Dans quelle mesure les prophéties se sont-elles réalisées? Et quelle en est notre lecture aujourd'hui? 


\section{La correspondance entre Anna et Sigmund Freud}

Il nous faut porter un regard d'abord sur la correspondance très touchante qui met en évidence, mais de façon discrète, le lien entre Anna et son père, à savoir la complicité, l'amour partagé et l'anticipation de ce que celui-ci demeurera, inchangé jusqu'à la mort de Freud et bien au-delà... Cela pourrait commencer ainsi: «Je dois tout de même t'écrire une fois de plus pour que tu ne m'oublies pas totalement pendant mon absence», lui écrit-elle de Merano ${ }^{4}$.

C'est à travers un rêve clairement œdipien de princesse qu'Anna s'adresse à son père: "J'ai récemment rêvé que tu étais un roi, et moi une princesse, et qu'on voulait nous dresser l'un contre l'autre par des intrigues politiques. Ce n'était pas beau et très énervant ${ }^{5}$. ${ }^{=}$Freud lui répond le lendemain: «Il est certain que les intrigues qui œuvraient à notre séparation ont échoué, il est possible que tu sois une princesse qui, comme dans le conte, doit désormais garder des oies, mais il est avéré que je suis désormais un roi sans terre. [...] Si Ischl est vraiment si coûteux [...] nous ne pourrons pas y tenir cour», plaisante-t-il, suite aux informations données par Anna sur le prix des auberges (Freud et Freud, 2012, p. 150-151).

Anna est alors une adolescente de 17 ans, elle est en villégiature, ou plutôt en cure thermale; ses parents s'inquiètent de son état de santé. Elle doit se reposer et prendre du poids, elle est aux prises avec des sentiments confus et intenses. Elle cherche sa place dans la famille et jalouse intensément sa sœur Sophie qui le lui rend bien, sœur qu'elle pense plus belle et plus douée qu'elle:

[Anna] admirait démesurément sa sœur Sophie en raison [...] de sa beauté et de sa féminité. Quand on connait les écrits d'Anna Freud, on est renvoyé pour ce qui concerne la relation qu'entretenaient ces deux sœurs au motif de la cession altruiste qu'elle a décrit bien plus tard comme l'une des formes de la défense du Moi et qui avait manifestement des racines dans l'observation du soi (Freud et Freud, 2012, p. 89, note 4).

Freud dans sa lettre à Anna du 5 janvier 1915 est alarmé de la voir si souffrante et lui propose une interprétation: son état ne serait dû à «aucun problème physique», mais à «la très ancienne jalousie envers Sophie dont tu n'es pas responsable, je le sais bien, mais elle beaucoup plus» (Freud et Freud, 2012, p. 88). On sent ici l'inquiétude paternelle qui va le conduire à la prendre en analyse dès 1918. 
Plus tard, Freud la rejoint à Venise pour un voyage "délicieux", écrit-il, alors qu'il avait prévu de rendre visite à Sophie, nouvellement mariée. Est-ce Venise, ville magique et symbole de l'amour, qui marque le moment tournant de sa préférence pour Anna?

Elle est bientôt la dernière à vivre à la maison avec ses parents et la tante Minna, et son statut et les rapports familiaux changent grandement. Freud l'appelle désormais «ma fille unique» (Gay, 1988, p. 495) et ma «chère et unique fille» dans une lettre du 21 juillet 1912 (Freud et Freud, 2012, p. 73). Maintenant que ses sœurs sont mariées, elle n'est plus la petite dernière plus ou moins acceptée de la famille. Elle s'occupe plutôt de tout le monde et de tout, par exemple des réservations d'auberges pour les vacances d'été et d'hiver, car la famille adore la forêt, la nature... Elle prend aussi soin des enfants de Sophie après sa mort, sœur dont elle était très jalouse jusqu'à son mariage; sa grande souffrance à ce sujet - entre autres - l'amène sur le divan.

C’est dire si Anna, la seule des trois filles à avoir accompli des études supérieures pour être institutrice et qui avait plusieurs vocations, n'avait plus le temps de continuer cette première carrière. Elle se consacre alors à sa famille, à son père, mais aussi à la psychanalyse, ce qui semble former pour elle un ensemble indissociable. Elle lisait par exemple aux congrès les communications de Freud, incluant celle sur la sexualité féminine, et participait donc à la (petite) politique inhérente aux conflits internes. Freud le souligne, elle s'occupe de tout, c'est-à-dire de l'essentiel et de ce qui rend la vie facile aux autres... Elle semble dévolue à cette mission. Dans ce contexte, elle devient psychanalyste à un très jeune âge, alliant et mêlant fondation, pédagogie novatrice et psychanalyse. Anna cherche ardemment à avoir une place parmi les grands, en l'occurrence les collègues de son père, et la meilleure place auprès de Freud.

Même si la correspondance s'attache surtout à une multitude de détails concrets et de questions d'intendance et d'organisation, ce qui en ressort plus largement est l'extrême complicité et l'amour entre Anna et son père. Freud y apparaît d'abord comme un bon père de famille, soucieux du bienêtre de ses enfants, de toute sa famille et de ses amis. On voit croître son attachement pour Anna au fil de la correspondance, jusqu'à l'aveu qu'il ne pourra pas se détacher d'elle si facilement et qu'elle est «très difficile à remplacer». Il s'attache à elle, «à son corps défendant», écrit-il à plusieurs de ses correspondants, et se débat avec l'éventualité peu probable de son futur mariage, tout en déclarant qu'elle est trop jeune et en éloignant les supposés prétendants, par exemple Jones dont il se méfie, craignant qu'il ne la lui 
arrache... (Gay, 1991, p. 497) Ainsi, il renonce petit à petit à l'idée qu'Anna trouve un mari et le quitte, comme ses sœurs avant elle; il finit par admettre sa grande dépendance envers elle. Par ailleurs, Anna elle-même ne manifeste aucun intérêt pour le projet de se marier...

Il semble bien, à lire la correspondance entre le père et la fille, qu'ils aient tous deux réalisé leur désir profond et que l'amour d'une mère pour son fils ne soit pas le seul véritable, contrairement à ce qu'avait déjà proposé Freud! Celui qu'il manifeste pour sa fille semble incommensurable.

C'est surprenant, mais on ne sent pas d'ambivalence dans cette relation œdipienne assumée. Au contraire, il y a tant d'élan et d'amour que c'en est touchant et troublant à la fois. Nul devoir ici ne semble les accabler ni l'un ni l'autre; au contraire, seul le plaisir se révèle. Aux derniers moments, Anna est encore et toujours présente aux côtés de son père. Selon Lucie Freud, «l'admirable dans les soins prodigués par Annerl ne fut pas son abnégation (au cours des dernières semaines, elle avait totalement perdu l'habitude de dormir) [...] L'admirable, c'est qu'il ne l'a jamais vue autrement qu'avec une expression de bonheur sur le visage» (Freud et Freud, 2012, p. 531).

Mais, avant qu'Anna soit au chevet de son père, Dorothy Burlingham et ses quatre enfants entrent dans sa vie pour toujours, ce qui semble plaire à Freud. Il parle ainsi de «la famille américaine» qui fait désormais partie de leur vie et qui partage leurs vacances; ils seront d'ailleurs aussi voisins au 19, rue Bergasse, à Vienne (Roudinesco, 2014, p. 342).

Après son analyse, Anna devient une jeune femme autonome et épanouie. Elle part notamment en voyage avec Dorothy Burlingham en Italie. «Heureuses et libres» (Roudinesco, 2014, p. 343), elles achètent une ferme à la campagne. En 1922, à moins de 27 ans, Anna est psychanalyste admise.

En 1923 toutefois, le diagnostic tombe: Freud est atteint d'un cancer de la mâchoire. Bien qu'elle poursuive ses projets de vie, de psychanalyste et de pédagogie avec Dorothy Burlingham, la vie d'Anna prend alors une autre voie. De 1923 jusqu'à la mort de Freud en 1939, Anna et lui ne se quittent presque plus. Et comme ils ne s'écrivent que quand ils sont séparés, la correspondance s'estompe. Selon Roudinesco, Anna renonce officiellement au mariage en 1923 (Roudinesco, 2012, p. 14). S'agirait-il d'une résolution partielle de la névrose de l'adolescente Anna, suite à son analyse et à sa rencontre avec Dorothy Burlingham et ses enfants? À la croisée des chemins, elle choisit sa compagne pour la vie, mais s'occupe aussi du corps de son père jusqu'à sa mort, ainsi que de son œuvre et de la sienne propre... 


\section{Analyse et sexualité}

La correspondance entre Sigmund et Anna Freud, très discrète, ne traite pas d'éléments pourtant essentiels et reliés, à savoir l'analyse d'Anna et la question de sa sexualité, oscillant entre fantasmes et rêveries masturbatoires, sublimation et ascétisme, amour filial et peut-être homosexuel.

L'analyse d'Anna avec son père, sans être inhabituelle à l'époque ni vraiment secrète, suscite un malaise. Les kleiniens en profitent pour la «discréditer» (Houssier, 2011, p. 17) et les freudiens préfèrent ne pas trop s'étendre sur le sujet, du moins jusqu'à la publication des biographies de Freud et d'Anna Freud (Gay, 1988; Young-Bruehl, 1988, p. 93-127). Il en va en quelque sorte comme de la lettre volée: en évidence, mais taboue. Pourtant les analyses d'enfants par leurs parents ou leurs proches sont monnaie courante, pour ne pas dire la norme. Ainsi Jung et Abraham ont analysé leurs enfants, Mélanie Klein les siens, ainsi que ceux de Jones à son arrivée à Londres (Young-Bruehl, 1988, p. 104; Fendrik, 1989, p. 10).

Répétition troublante du scénario et révélatrice des méthodes de l'époque, Anna Freud reçoit elle-même en analyse les enfants de Dorothy Burlingham, Erik Erikson alors jeune précepteur à l'école et son neveu Ernst:

Nous étions tous en analyse avec A. F.: Tinky, Bob, Michael, Erik Homberger-Erikson, Ernsti Halberstadt-Freud et moi [...] Au lieu d'observer la séparation de la sphère thérapeutique et de la sphère privée postulée par les freudiens, A. F. tissait en toute innocence la toile d'araignée où un si grand nombre d'entre nous, bénéficiaires et victimes, restèrent piégés. (Heller, 1996, p. 24)

À une lettre d'Edouardo Weiss qui pense analyser son fils, Freud répond en 1935 (ne remettant donc pas en cause cette cure et sa validité) que c'est «une affaire délicate» pour les fils, mais qu'«avec ma propre fille, j’ai bien réussi» (Young-Bruehl, 1988, p. 103).

En deux tranches, l'analyse d'Anna par son père dure de 1918 à 1920 puis de 1922 à 1924, mais peut-être un peu entre ces deux périodes et par la suite aussi (Gay, 1991, p. 504; Young-Bruehl, 1991, p. 93127; Rodrigué, 1996b, p. 219-221; Faure-Pragier, 1997, p. 139; Houssier, 2011, p. 1920). Freud est inquiet des symptômes persistants d'Anna et il n'est prêt à la confier à aucun analyste de son entourage, d'abord parce qu'ils sont loin d'eux (à Berlin ou à Budapest), ensuite parce qu'il préfère ne pas se séparer d'elle ni prendre de risque et, enfin, pour des raisons financières (Young-Bruehl, 1988, p. 117). 
La névrose d'Anna tourne autour de fantasmes de fustigation apparus à l'âge de cinq à six ans, stimulés par une masturbation compulsive, puis de rêveries qu'elle appelle «belles histoires» qui l'absorbent beaucoup trop et dont elle aimerait se débarrasser. Lorsque ces fantasmes violents qui lui font peur resurgissent en 1922, elle reprend l'analyse. Elle découvre par la suite leur parenté avec les «belles histoires» apparues entre huit et dix ans, soit une autre forme de fantasmes stéréotypés et enjolivés, préfigurant une sublimation qui aurait réussi (Young-Bruehl, 1988, p. 94).

L'analyse a fait l'objet d'au moins une des vignettes cliniques de l'article «Un enfant est battu» de Sigmund Freud (1919) et de la présentation (1922) prononcée par Anna Freud le 31 mai 1922 en vue de devenir membre de la WPV (Roudinesco, 2012, p. 14). Elle avait alors 26 ans et ne voyait pas encore officiellement de patients: "Anna, comme son père, exposa son cas comme s'il se fut agi d'une autre personne.» (Rodrigué, 1996b, p. 222)

Pendant une période, Lou Andreas Salomé (qui a le même âge que Martha) est invitée par Freud comme tiers pour soutenir Anna, dans une relation à la fois amicale, maternelle et analytique. Elle recueille ses confidences et associations, notamment sur son analyse où le père et l'analyste ne font qu'un. Il manquerait en effet un troisième terme: «Nos soirées d'analyse sont quelque chose de très étrange, tout particulièrement parce que la troisième personne manque, celle qui est utilisée dans le transfert et envers qui les conflits sont exprimés et résolus », lui confie-t-elle avec perspicacité le $1^{\text {er }}$ juin 1924 (Bucher, 2003, p. 176).

Plus tard, Anna s'éloigne de l'analyse et de Lou et se lie à Eitingon, puis à Eva Rosenfeld, qui, comme Dorothy Burlingham, est en analyse avec Freud. Elle lui écrit: «il n'y a aucune contradiction dans le fait que tu suives une analyse là où tu préférerais seulement aimer. J'ai fait de même et, peut-être pour cette raison, les deux choses sont devenues pour moi inextricablement liées.» (Freud, 1992, p. 144)

Aimer et analyser vont de pair: les enfants sont analysés par leurs parents ou leurs proches et les trois amies se retrouvent toutes sur le divan de Freud. Cette promiscuité est surprenante et déstabilisante aujourd'hui. Pourtant, pour Florian Houssier, ces croisements se situent dans le contexte de l'époque, mais aussi dans celui d'une «famille élargie» pour Anna comme pour Freud, famille «au sein de laquelle, comme avec son père, le mélange entre psychanalyse (des enfants) et vie personnelle est une constante» (Houssier, 2011, p. 18).

En outre, il est clair que pour Anna Freud, pédagogie et psychanalyse sont intimement liées, comme en témoigne sa pratique dans laquelle la 
psychanalyse des enfants qui fréquentent les écoles à pédagogie nouvelle va de soi; les deux versants - thérapeutique et éducatif - se complètent.

Cette situation, où une jeune fille est analysée par son père, avec publications et cooptation institutionnelle à la clé, et qui aboutit à une résolution de la sexualité sous la forme d'une sublimation ascétique, apparaît de nos jours comme troublante et quelque peu tragique. À ce propos, Émilio Rodrigué déclare sans ambages: "C'était un acte complètement irrégulier. Ce fut la plus grande transgression dans la vie de Freud» (Rodrigué, 1996b, p. 219). Pourtant, la découverte de la psychanalyse et de l'inconscient a suscité à l'époque ce genre de situations où se mêlent les interlocuteurs et les rôles, d'une manière qui nous apparaît incestueuse, mais qui ne semblait pas comme telle à l'époque: il s'agissait de se «libérer» d'une répression de la sexualité «refoulée» et donc vue comme pathogène.

\section{Impossible séparation avec le père, désir sexuel et sublimation}

Freud est attristé de ce à quoi il ne peut se dérober: il n'est pas satisfait de l'«orientation générale» de la vie d'Anna. Il manifeste notamment son inquiétude quant à son «refoulement sexuel» dans une lettre à sa «chère Lou», le 10 mai 1925: «L'analyse d'Anna continue [...] Elle fait de grands progrès [...] J'ai peur que son refoulement sexuel ne lui joue un mauvais tour. Je ne peux rien pour la libérer de moi et personne ne m'y aide.» (Gay, 1991, p. 506)

Toutefois, Freud n'est pas dupe: il a besoin d'Anna sur un mode addictif, comme d'un cigare dit-il. Seule la mort peut les séparer:

Si elle devait vraiment s'en aller, je me sentirais aussi appauvri que je le suis en ce moment $[\ldots]$ ou que s'il me fallait renoncer à fumer. [...] À cause de tous ces conflits insolubles, il est bon que la vie prenne fin quelque jour. (Lettre à Lou du 13 mars 1922, citée dans Gay, 1988, p. 506 et YoungBruehl, 1988, p. 106)

Il ne peut pas renoncer à elle et il craint que cela n'accentue sa tendance au refoulement, ou plutôt au renoncement. Pour ne pas perdre Freud, Anna aurait-elle sacrifié sa sexualité et sublimé ses pulsions grâce à la «cession altruiste», déjà pratiquée avec Sophie?

La thèse de la sublimation et de l'ascétisme d'Anna prévaut dès les débuts de l'analyse et s'associe à un rôle masculin. C'est ce qui ressort du texte qu'elle présente à la WPV le 31 mai 1922 en vue de son admission: 
La sublimation de l'amour sensuel (de la fille pour son père) en amitié pleine de tendresse est bien sûr facilitée par le fait que dès les premiers stades du fantasme de fustigation la fillette a abandonné les différences entre les sexes et apparaît systématiquement sous les traits d'un garçon. (Young-Bruehl, 1988, p. 98)

Quant à la "cession altruiste», c'est la manière dont Anna se débarrasse de ses fantasmes de fustigation, embellis et devenus de «belles histoires», néanmoins obsédantes. Cette sublimation sous forme de renoncement et d'austérité semble faire l'objet d'une décision consciente et délibérée. Seraitelle le prix du compromis par rapport au désir incestueux? Voici ce qu'elle dit du cas d'une jeune fille jalouse de sa sœur dans un fragment de son auto-analyse:

Elle tolère très bien chez les autres certaines pulsions instinctuelles que son surmoi condamnait quand elles affectaient son propre moi et elle satisfait ses instincts en participant à la satisfaction instinctuelle de son prochain, ce que lui permettent les mécanismes de la projection et de l'identification. [...] Toute sa conduite est ainsi caractérisée par ce transfert à d'autres de ses propres désirs [...] elle a retrouvé sa gaieté et totalement oublié que c'est à sa sœur et non à elle-même que le plaisir échoit. (Freud, 1936, p. 116-117)

Une fois le «choc» de l' «humiliante déception» dépassé grâce à l'analyse, le renoncement altruiste - et égoïste, dit-elle - lui apporte le plaisir qu'elle ne peut obtenir avec le jeune homme qui courtise la sœur plutôt qu'elle-même...

Elle ne semble pas attirée par les hommes, malgré des amitiés intenses, par exemple avec Eitingon après la fin de son analyse, non sans une grande déception d'ailleurs. Son désir, dans lequel elle serait identifiée à un garçon, irait plutôt du côté féminin. En 1922, Anna écrit à Lou:

Pour la première fois j'ai eu un rêve diurne où apparaissait un protagoniste féminin. C'était même une histoire d'amour à laquelle je n'ai pas cessé de penser. Je voulais tout de suite l'exploiter et l'écrire mais papa a trouvé que je ferais mieux de la laisser tomber et de penser à mon exposé. (Roudinesco, 2012, p. 14) 
Rêverie et projet d'écriture préfigurent un désir homosexuel, ce qui inquiéterait Freud, qui lui demande d'y renoncer, selon Anna. Freud avaitil peur que sa fille ne devienne lesbienne? En tout cas, il est pris dans son dilemme: il ne veut plus vraiment qu'elle se marie - l'a-t-il jamais vraiment souhaité? -, et elle non plus d'ailleurs. Mais il aime beaucoup la «famille américaine» avec qui sa fille est en «symbiose», et il entend sur le divan parler d'Anna par Dorothy Burlingham arrivée à Vienne en 1925 pour entreprendre une analyse. Il commence à comprendre les sentiments qui les relient et qu'Anna lui cache, comme elle les cache à Lou. (Young-Bruehl, 1988, p. 124)

Alors, ces liens, quels sont-ils? Sublimés et platoniques, ou amoureux et sexuels? Il est difficile d'y répondre. Mais est-ce vraiment intéressant ou pertinent? Ils sont déniés par Anna Freud d'abord et par la communauté analytique ensuite. Ils sont donc secrets et frappés d'un interdit de dire, sinon par le biais de dénégations, d'allusions ou même de médisances... Ainsi Élisabeth Roudinesco peut écrire dans un premier temps qu'elles sont comme des jumelles et qu'elles ne sont surtout pas lesbiennes puisqu'elles considèrent «l'homosexualité comme une maladie». Ce sont les propos des «mauvaises langues»:

Leur amitié était si intense qu'elles se regardèrent bientôt comme des sœurs jumelles et finirent par se ressembler physiquement. Cette amitié sembla suspecte, et quelques mauvaises langues les accusèrent d'être des lesbiennes, ce qui, pour Anna, était l'injure suprême. Elle considérait en effet l'homosexualité comme une maladie, contrairement à son père. (Roudinesco et Plon, 1997, p. 167)

Dans un deuxième temps, Elisabeth Roudinesco parle des effets mixtes ou mitigés de l'analyse d'Anna puisqu'elle en est réduite à «haïr sa propre homosexualité»: «Si la cure d'Anna par son père lui permet de s'affirmer comme un chef d'École entouré des meilleurs disciples de celui-ci au sein du Kinderseminar, elle aura pour conséquence néfaste de lui faire haïr sa propre homosexualité» (Roudinesco, 2014, p. 15). Est-ce le prix à payer pour être dans le cercle des élus et des fondateurs?

Elizabeth Young-Bruehl reprend à son compte l'hypothèse de la sublimation plutôt que celle du refoulement:

Elle avait l'énergie impressionnante, quelque peu compulsive, qui caractérise les êtres chastes habités par une foi ardente ou les causes irrésistibles. 
Ses conflits n'étaient pas non plus résolus. Elle pouvait manifester un intérêt scientifique pour la sexualité, sans avoir d'activité ni homosexuelle ni hétérosexuelle. [...] sa défense principale était la sublimation et donc: pas le refoulement. (Young-Bruehl, 1988, p. 434)

«Lesbiennes», «sœurs», «jumelles»: décidément, il semble difficile de décrire le lien entre Anna et Éva Rosenfeld (qui a précédé Dorothy dans la vie et le cour d'Anna) et entre Anna et Dorothy Burlingham (qui s'établira par la suite pour le reste de sa vie). Ce qui semble par contre clair et intéressant, c'est que ce projet de vie et d'amitié a existé bien avant la mort de Freud et qu'il est donc inexact de dire qu'Anna ne vivait que pour son père et n'aimait que lui. Au-delà de sa fidélité légendaire à Freud et à son œuvre, elle a certainement eu une vie affective et sentimentale après son analyse, comme elle l'écrit en conclusion du chapitre «Une forme d'altruisme»: la «jeune institutrice», qui pourrait bien une version d'être elle-même, a pu réaliser «sous une forme, il est vrai, sublimée, [...] certains désirs instinctuels que l'analyse lui a permis de réintégrer dans sa propre vie» (Freud, 1936, p. 124).

\section{Fondations, amitiés et famille psychanalytique}

Les Lettres à Eva Rosenfeld montrent une Anna enjouée, affectueuse, aimante, dévouée et légère. Elle adresse ses lettres à «Eva chérie» ou à «ma chère Eva», et les finit par "ton Anna", "c'est un vrai méli-mélo mais c'est un petit bout de moi pour toi» (Freud, 1992, p. 212), ou encore «je t'embrasse tous les jours de l'année avec la même tendresse» (Freud, 1992, p. 222). Cette amitié intense et fusionnelle - on pourrait même dire «amoureuse» - se joue entre les trois protagonistes qui fondent l'École Hietzing à Vienne (1927-1932), puis les Jackson Nurseries en 1937 avec Peter Blos et Erik Erikson. La vocation principale du trio féminin - avec leurs alliés - est un projet psychanalytique de pédagogie progressiste de vie, dans lequel elles s'engagent totalement: Eva Rosenfeld offre sa maison (son fils Victor fait d'ailleurs partie des enfants de l'école) et Dorothy Burlingham finance le projet, conçu d'abord pour ses quatre enfants, auxquels se joignent, entre autres, ceux d'une famille américaine amie (les Sweester), Ernst Halberstadt (surnommé «l'enfant à la bobine» [Roudinesco, 2014, p. 19], neveu d'Anna Freud, protégé et fils spirituel) et Peter Heller (Heller, 1996, p. 24). Anna Freud est pédagogue, analyste et presque mère des enfants de sa compagne et des autres. Elle aurait ainsi réalisé en partie son désir écrit-elle à Eitingon: «Je pense que je veux non seulement les guérir, mais aussi, en même temps 
les avoir à moi, ou avoir quelque chose d'eux à moi [...] Vis-à-vis de la mère des enfants, les choses ne sont pas très différentes.» (Young-Bruel, 1988, p. 121) Elles sont toutes trois en analyse avec Freud, quoiqu'Anna ne le soit plus quand les autres entreprennent la leur sur son invitation et celle de Freud, qui prend Eva gracieusement. Au fur et à mesure que le lien avec Dorothy se développe et se confirme, la relation fusionnelle entre Anna et Eva est de plus en plus entachée par la jalousie de cette dernière à l'égard de Dorothy. La division survient finalement quand Eva Rosenfeld les précède à Londres en 1937 et commet l'ultime trahison: elle reprend son analyse avec Melanie Klein. Cela confirmera le froid dans la relation, sans qu'il n'y ait de véritable rupture.

Peter Heller a raconté son analyse avec Anna Freud et la vie à l'école avec les enfants Burlingham. Plus tard, il épouse Catherine (surnommée «Tinky»), la plus jeune des filles de Dorothy Burlingham, qui devient ainsi sa belle-mère, puis ils divorcent. Heller donne d'elle et de ses relations avec la famille Freud, les enfants et la psychanalyse, une image peu flatteuse, mais révélatrice de l'ambiance de l'époque et probablement de son propre rapport à elle. Surnommée «mother» par les enfants de l'école, "[Dorothy] se transformait en vassale complètement soumise et pleine de zèle religieux devant le Professeur et Anna Freud». Il ajoute, par rapport à l'institution psychanalytique:

En toute innocence, elle en vint à représenter cette orthodoxie étriquée de cœur et d'esprit, cette prétention de tout savoir, ce souci mesquin de la hiérarchie et cet esprit de chapelle vindicatif qui rendaient les freudiens tellement insupportables. (Heller, 1996, p. 22-23)

Il commente ainsi le portrait de Dorothy proposé par Elizabeth YoungBruehl qui, selon lui,

diffère considérablement des nombreux souvenirs que je garde de cette femme. En dépit de son apparence fragile, c'était une matrone énergique et agressive, dotée d'une ténacité peu commune. Fanatique dans son dévouement à Anna et Sigmund Freud, elle était aussi éprise de moralité, de grandeur d'âme et de générosité qu'elle était intolérante, satisfaite d'elle-même et intransigeante - conséquence sans doute de son orgueil puritain et patricien. (Heller, 2003, p. 93-94) 
Ce commentaire plutôt décapant réfère à l'autre face de la médaille, à savoir celle d'une rigidité et d'une fermeture, ce qui n'empêche pas Peter Heller, comme ancien élève nostalgique, de vanter l'école, «l'ayant appréciée et (s') étant épris au point de les adorer, du style Burlingham, de l'aisance libératrice qui y régnaient» (Heller, 2003, p. 103).

Quant à Ernst, l'enfant du fort/da et protégé d'Anna Freud: «Freud avait fait de lui un portrait saisissant dans Au-delà du principe de plaisir: celui d'un enfant de six ans aimé de ses parents, qui jetait et ramenait à lui une bobine en criant “fort/da".» (Roudinesco, 2012, p. 16) Il a suivi les voies de la transmission psychanalytique familiale des Freud, de Sigmund à Anna, démontrant ainsi sa filiation à celle qui l'a accompagné et relié à l'histoire familiale de la psychanalyse.

En quête d'une identité qui pût le rattacher à son grand-père, il se fit appeler Ernst W. Freud pour ne pas être confondu avec son oncle. À la mort d'Anna, il s'en alla exercer la psychanalyse en Allemagne, renouant ainsi, pour la pratiquer, avec la langue de son enfance. Il fut le seul descendant mâle de la famille Freud à devenir psychanalyste. (Roudinesco, 2014, p. 574)

\section{Conclusion}

Aujourd'hui, le ton et les temps changent; la polémique et les controverses sont moins aiguës et le contexte n'est plus le même. Il n'est plus aussi essentiel de soutenir et de justifier des positions orthodoxes, notamment sur l'homosexualité, et de montrer patte blanche. Probablement et tristement, ces prises de position rigides et sérieuses ont été prônées par Anna Freud et Dorothy Burlingham elles-mêmes, d'une manière défensive.

Anna Freud a certainement été fidèle à la mémoire et à l'œuvre de son père et à ce qu'elle a cherché à en protéger. Elle a tout de même eu une longue vie après la mort de Freud. Elle a aimé, fondé, créé et frayé son propre chemin. Qu'elle ait été lesbienne dans son corps ou avec d'autres personnes, hommes ou femmes d'ailleurs, par sublimation ascétique ou cession altruiste ou dans des actes en secret, cela importe peu finalement et ne nous concerne pas. Comme dit Peter Heller, la psychanalyse nous apprend que la sexualité n'est pas qu'une question de corps...

Plutôt qu'un couple, Anna et Dorothy Burlingham constituaient une famille dans la grande famille de Freud et des enfants en analyse avec des psychanalystes. Pourrait-on dire, à la suite de Silvia Fendrik, que la psychanalyse d'enfants à ses débuts était "celle d'un espace clinique nouveau fournissant la possibilité de séparer les enfants-patients de leurs parents-analystes»? 
(Fendrik, 1989, p. 11) Quant à la pratique familiale en vase clos de la psychanalyse et ses effets et débordements, on peut bien en être averti aujourd'hui, mais «cela n'empêche pas d'exister», ni d'en vivre les effets. On essaie de s'en débrouiller comme on peut. Encore faut-il renouer avec cette histoire qu'on porte et perpétue dans sa méconnaissance et l'analyser sans trop s'en indigner, mais plutôt avec légèreté, ouverture et affection.

\author{
Marie Hazan \\ hazan.marie@uqam.ca
}

\title{
Notes
}

1. Selon le petit-fils de Dorothy Burlingham, Peter Heller lui aurait parlé du rapport d'Anna Freud avec la sublimation: "She was very strong when it came to demanding and furthering sublimation $»$. Il raconte aussi l'anecdote suivante: Eduard Hirtschmann aurait fait un commentaire ironique selon lequel, alors que Freud parlait des pulsions, Anna enseignait les défenses... (Burlingham, 1989, p. 210)

2. Voir la très belle pièce Le visiteur d'Éric Emmanuel Schmitt (Acte Sud, 1993) où Freud, bien qu'athée, est prêt à tout pour voir son Anna échapper à la Gestapo, jusqu'à (se) débattre avec Dieu en attendant sa libération...

3. En note de bas de page au rêve du 23-24 juillet 1895, Freud fait en effet allusion à une troisième personne, qui s'avère être sa propre femme, et qui était «bouffie» et «résistait» à l'examen (Freud, 1900, p. 102).

4. Lettre du 26 novembre 1912 (Freud et Freud, 2012, p. 76). Merano est une station thermale du Tyrol et qui faisait alors partie de l'Autriche-Hongrie; elle est italienne depuis la Première Guerre mondiale.

5. Lettre du 6 août 1915 (Freud et Freud, 2012, p. 150-151). Selon la traduction d'Emilio Rodrigué (1996 a, p. 218) : «C’était déplaisant, même bouleversant ».

\section{Références}

Anzieu, D. (1959). L'auto-analyse de Freud. Paris: Presses universitaires de France, 1992.

Burlingham, M. J. (1989). The last Tiffany. New York: Atheneum.

Bucher, J-P. (2003). Anna Freud, quel passage à l'analyste? (1921-1925). Essaim, 2003-1 (11), 171-178.

Drillon, J. (2012). Mademoiselle Anna Freud. Le Nouvel Observateur. Repéré à http://bibliobs. nouvelobs.com/documents/20121031.OBS7585/mademoiselle-anna-freud.html

Faure-Pragier, S. (1997). L'influence d'Anna sur la théorie freudienne. Dans Les bébés de l'inconscient (p. 130-141). Paris: Presses universitaires de France.

Fendrik, S. (1989). Fiction des origines de la psychanalyse avec les enfants. Paris: Denoël.

Freud, A. (1922). Fantasme d' «être battu» et rêverie. Dans M.-C. Hamon (dir.), Féminité mascarade (p. 57-75). Paris: Seuil, 1994.

Freud, A. (1936). Le Moi et les mécanismes de défense. Paris: Presses universitaires de France, 1949.

Freud, A. (1992). Lettres à Eva Rosenfeld. 1919-1937. Paris: Hachette, 2003.

Freud, A., Freud, S. (2012). Correspondance Sigmund Freud, Anna Freud. 1904-1938. Paris: Fayard.

Freud, S. (1900). L'interprétation des rêves. Paris: Presses universitaires de France, 1967. 
Freud, S. (1919). Un enfant est battu. Dans Névrose psychose et perversion (p. 219-243). Paris: Presses universitaires de France, 1973.

Freud, S. (1956). La naissance de la psychanalyse, 1887-1904. Suivi de L'esquisse d'une psychologie scientifique. Paris: Presses universitaires de France.

Gay, P. (1988). Freud, une vie. Paris: Hachette 1991.

Gottwaldt, A. (2004). Les sœurs de Sigmund Freud et la mort. Remarques concernant leur destin de déportation et de meurtre de masse. Revue française de psychanalyse, 68, 1307-1316.

Heller, P. (1983). Une analyse d'enfant avec Anna Freud. Paris: Presses universitaires de France, 1996.

Heller, P. (2003). Le contexte et les thèmes majeurs des lettres. Dans A. Freud, Lettres à Eva Rosenfeld. 1919-1937. Paris: Hachette.

Houssier, F. (2011). Sigmund Freud et son Antigone: adolescence et liens de mutualité théoriques. Topique, 2011/2 (115), 17-32.

Menahem, R. (2003). Désorientations sexuelles. Revue française de psychanalyse, 67, 11-25.

Réfabert, P. (2004). La théorie de l'hystérie grevée par la carence d'un témoin. Dans Comme si de rien: témoignage et psychanalyse. Paris: Campagne Première, 2018.

Rodrigué, E. (1996a). Freud Le siècle de la psychanalyse, tomes 1 et 2. Paris: 2000.

Rodrigué, E. (1996b). Anna, la jumelle de la psychanalyse. Dans Freud, le siècle de la psychanalyse, tome 2. Paris: Payot, 2000.

Roudinesco, E., Plon, M. (1997). Dictionnaire de la psychanalyse. Paris, Fayard 1997.

Roudinesco, E. (2012). Préface. Dans A. Freud et S. Freud, Correspondance Sigmund Freud, Anna Freud. 1904-1938. Paris: Fayard.

Roudinesco, E. (2014). Sigmund Freud en son temps et dans le nôtre. Paris: Seuil.

Sartre, J.-P. (1984). Le scénario Freud. Paris: Gallimard.

Schneider, M. (1980). Blessures de mémoire. Paris: Gallimard 1980

Young-Bruehl, E. (1988). Anna Freud. Paris: Payot 2006. 